\title{
Long-term outcomes following adjuvant endocrine therapy in breast cancer patients with a positive-to-negative change of hormone receptor status following neoadjuvant chemotherapy
}

\author{
JIA YI WU*, WEI GUO CHEN*, XIAO SONG CHEN, OU HUANG, \\ JIAN RONG HE, LI ZHU, YAFEN LI and KUN WEI SHEN \\ Comprehensive Breast Health Center, Ruijin Hospital, Shanghai Jiaotong University \\ School of Medicine, Shanghai 200025, P.R. China \\ Received May 27, 2014; Accepted July 2, 2014
}

DOI: $10.3892 / \mathrm{mco} .2014 .365$

\begin{abstract}
The aim of the present study was to investigate whether breast cancer patients with changes from positive to negative in the hormone receptor following neoadjuvant chemotherapy (NAC) could benefit from adjuvant endocrine therapy (ET). Between December 2000 and November 2010, 97 eligible patients with a positive-to-negative switch of the hormone receptor status following NAC were identified. All the patients were categorized into two groups on the basis of the administration of ET: 57 ET-administered and 40 ET-naïve patients. Survival analyses were performed to examine the prognostic value of ET administration, as well as other clinical and pathological variables. The administration of ET was associated with a significantly improved disease-free survival (DFS) $(\mathrm{P}=0.018)$ in patients with a positive-to-negative switch of the hormone receptor status. The 5-year DFS rates were 77.0 and $55.5 \%$ in ET-administered and ET-naïve patients, respectively. The 5-year overall survival (OS) rate for ET-administered was also higher than that of the ET-naïve patients ( 81.3 vs. $72.7 \%, \mathrm{P}=0.053)$, but the difference between the two groups did not reach a statistical significance. The present study revealed that patients with the hormone receptor that was altered from positive to negative following NAC benefit from ET, and the hormone receptor status should be evaluated not only in specimens obtained during post-NAC surgery, but also in specimens biopsied prior to NAC.
\end{abstract}

Correspondence to: Professor Kun Wei Shen, Comprehensive Breast Health Center, Ruijin Hospital, Shanghai Jiaotong University School of Medicine, 197 Ruijin Second Road, Shanghai 200025, P.R. China

E-mail:kwshen@medmail.com.cn

*Contributed equally

Key words: breast cancer, endocrine therapy, neoadjuvant chemotherapy, hormone receptor

\section{Introduction}

The reliable determination of predictive factors of breast cancer is a prerequisite for selection of optimal therapeutic strategy. Estrogen receptor (ER) and progesterone receptor are currently the established tumor markers guiding routine use of endocrine therapy (ET). Neoadjuvant chemotherapy (NAC) is the standard treatment in the early years for patients with locally advanced breast cancer, and thus far, it is an increasingly used option for operable breast cancer $(1,2)$. Certain studies have reported that the ER and progesterone receptor status may alter during the course of NAC (3-6). However, limited studies have been performed regarding whether patients with a hormone receptor change from positive to negative following NAC could gain any benefit from adjuvant ET. The present study aimed to determine whether ET could provide any benefit in this subpopulation of patients with breast cancer.

\section{Materials and methods}

Patients. A total of 97 out of 687 (14.1\%) primary breast cancer patients who were treated with NAC at the Comprehensive Breast Health Center (Ruijin Hospital, Shanghai, China) between December 2000 and November 2010 were retrospectively collected. The eligibility criteria included female unilateral breast cancer patients treated with $\geq 3$ and $\leq 6$ cycles of NAC. The following data were also required: Age, menopausal status, clinical tumor size and lymph node status, pathological axillary lymph node involvement and follow-up information. Only the patients with a positive-to-negative switch in the hormone receptor status following NAC were included in the study. All the patients were classified into two groups on the basis of ET administration: 57 ET-administered and 40 ET-naïve patients.

Treatment modalities. For the majority of patients, NAC treatment was supplemented with one of the following three protocols: $\mathrm{NE}\left(25 \mathrm{mg} / \mathrm{m}^{2}\right.$ vinorelbine on day 1 and day 8 plus $60 \mathrm{mg} / \mathrm{m}^{2}$ epirubicin on day 1 , every 21 days), CEF $\left(500 \mathrm{mg} / \mathrm{m}^{2}\right.$ cyclophosphamide on day $1,75 \mathrm{mg} / \mathrm{m}^{2}$ epirubicin on day 1 and $500 \mathrm{mg} / \mathrm{m}^{2}$ fluorouracil on day 1 , every 21 days) and ED 
( $75 \mathrm{mg} / \mathrm{m}^{2}$ epirubicin on day 1 plus $75 \mathrm{mg} / \mathrm{m}^{2}$ docetaxel on day 1 , every 21 days). The other NAC regimens included $\mathrm{PCb}$ (paclitaxel and carboplatin) and DCb (docetaxel and carboplatin).

All the patients underwent radical surgery. Adjuvant chemotherapy was administered following surgery, and a total of 6-8 courses of chemotherapy were completed according to the preference of the physician treating the patient on the basis of the clinical and pathological findings following the surgery. Radiotherapy was applied subsequent to the completion of the adjuvant chemotherapy. The prescription of ET and the choice of the specific drugs were also determined according to the physician and/or the patient's preferences. Only one patient with human epidermal growth factor receptor 2 (HER2)-positive disease received trastuzumab.

Assessment of response. The clinical assessment of the response was based on the clinical measurements in the longest diameter of the tumor and node according to the Response Evaluation Criteria in Solid Tumors (RECIST) 1.1 (7) and was classified as follows: Complete response (CR), the disappearance of the disease; partial response (PR), $\geq 30 \%$ decrease; progressive disease (PD), $\geq 20 \%$ increase in the sum of the longest diameter of the target lesions or the appearance of new lesions; and stable disease (SD), neither sufficient shrinkage to qualify for PR or a sufficient increase to qualify for PD. The pathological complete response (pCR) was defined as the absence of invasive tumor in the breast and axillary lymph node. The patients with pCR following NAC were excluded from the analysis, as the hormone receptor status subsequent to surgery could not be accurately evaluated.

Hormone receptor and HER2 status determination. All the patients underwent a 14-gauge core needle biopsy (CNB) prior to NAC. The ER, progesterone receptor and HER2 status of $\mathrm{CNB}$ and the surgical specimens were determined by immunohistochemistry (IHC). Positive staining for ER/progesterone receptor was defined as nuclear staining in $\geq 10 \%$ of the tumor cells. The hormone receptor was defined as positive if the ER and/or progesterone receptor were positive and as negative if the ER and progesterone receptor were negative. HER2 positivity was considered as HER2 $3+$ by IHC or positive on fluorescence in situ hybridization (FISH), whereas cases with $0-1+$ or $2+$ without FISH detecting were regarded as negative. The following antibodies were used for the IHC test: Anti-ER, clone 1D5 (rabbit monoclonal; Gene Corp., Capinteria, CA, USA); progesterone receptor, clone PR636 (mouse monoclonal; Dako, Carpinteria, CA, USA); and HER2, c-erbB-2 (2000-2008, rabbit polyclonal; Dako) or 4B5 (2009-2010, rabbit monoclonal; Roche, Basel, Switzerland).

Statistical analysis. The $\chi^{2}$ test was applied to evaluate the association between the administration of ET and the other parameters studied. Fisher's exact test was performed when necessary. The disease-free survival (DFS) interval was defined as the time from the date of the first administration of NAC to the earliest occurrence of all the local, regional or distant recurrences, all the second cancers and contralateral breast cancers, and all the fatalities. The overall survival (OS) was defined as the time from the date of the first administration of NAC to all the mortalities, whether they were breast cancer-related or not. DFS and OS were estimated using the Kaplan-Meier analysis and the survival curves were compared using the log-rank test. Multivariate Cox regression analysis with stepwise selection was used to estimate the hazard ratio (HR), 95\% confidence interval (CI) and the effects of the clinical and pathological variables. All the statistical tests were two-sided and $\mathrm{P}<0.05$ was considered to indicate a statistically significant difference. The software package SPSS 16.0 for Windows XP (SPSS, Inc., Chicago, IL, USA) was used for analysis.

\section{Results}

Patient characteristics and treatment. A total of 97 eligible patients without $\mathrm{pCR}$ following NAC were identified to have a positive-to-negative change in the hormone receptor status. The median age was 51 years (range, 31-74 years) and $55.7 \%$ of these patients were premenopausal. A total of 30 patients (30.9\%) had stage IIb disease, 37 (38.1\%) exhibited stage IIIa disease and the remaining patients (30.9\%) had stage IIIb-IIIc.

The majority of the patients received one of the following three NAC regimens: NE (38.1\%), CEF (36.1\%) and ED (14.4\%). The remaining patients received either the $\mathrm{PCb}(7.2 \%)$ or $\mathrm{DCb}$ (4.1\%) regimen. As for the clinical tumor response according to RECIST 1.1, CR was documented in 20 patients (20.6\%), $53(54.6 \%)$ obtained PR, SD was observed in 21 (21.6\%) and three (3.1\%) had PD.

A total of 57 patients $(58.8 \%)$ received ET (47 with tamoxifen only, 39 with aromatase inhibitors only and 11 with tamoxifen and aromatase inhibitors). The correlation between the characteristics of the patients and ET administration are summarized in Table I. The patients treated with or without ET did not differ significantly in their clinical or pathological characteristics.

Hormone receptor status and HER2 status. A total of 77 patients (79.4\%) had ER-positive/progesterone receptor-positive tumors, 14 (14.4\%) had ER-positive/progesterone receptor-negative tumors and six (6.2\%) exhibited ER-negative/progesterone receptor-negative tumors. A positive pre- and post-NAC HER2 status was observed in 10 (10.3\%) and 11 patients (11.3\%), respectively. Two patients had a positive-switch of HER2 status following NAC and a negative-switch of HER2 status was observed in one patient. The pre-NAC ER/progesterone receptor status and pre/post-NAC HER2 status are shown in Table II.

DFS and OS. After a median follow-up of 68 months (range, 14-103 months), the overall DFS and OS rates were 61.9 and $70.1 \%$, respectively. The Kaplan-Meier curves for DFS in the two groups are shown in Fig. 1. The differences between the two curves were statistically significant, as determined by the log-rank test $(\mathrm{P}=0.018)$. The 5-year DFS rates in ET-administered and ET-naïve patients were 77.0 and 55.5\%, respectively. The Kaplan-Meier curves for OS are shown in Fig. 2. The 5-year OS rate for ET-administered was higher than that of the ET-naïve patients ( 81.3 vs. $72.7 \%, \mathrm{P}=0.053$ ), but the difference between the two groups did not reach statistical significance. In the exploratory subgroup analysis according to 


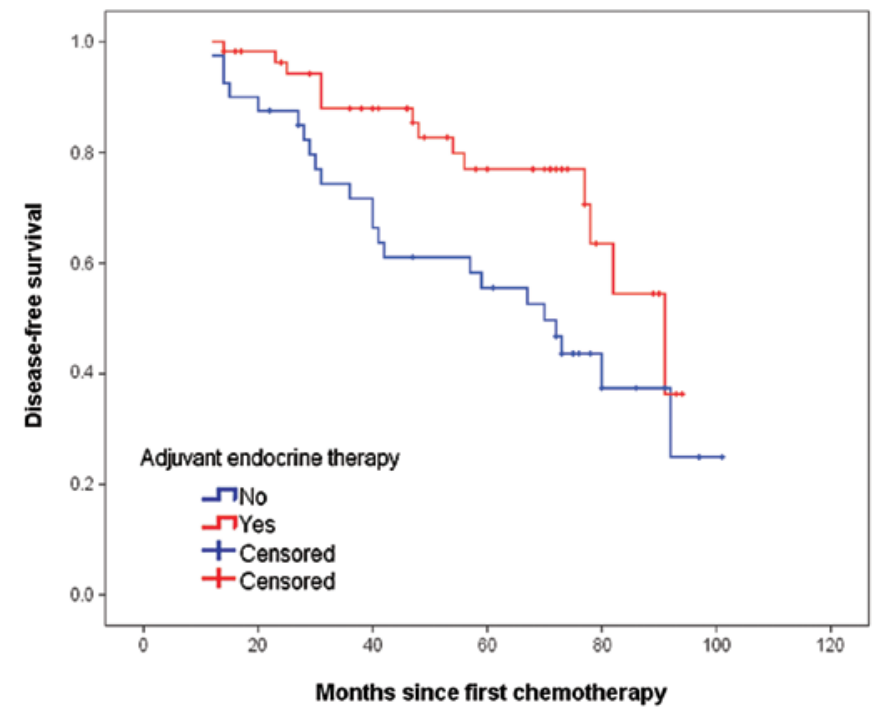

Figure 1. Correlation between the use of ET and DFS in all the patients $(\mathrm{P}=0.018)$. ET, endocrine therapy; DFS, disease-free survival.

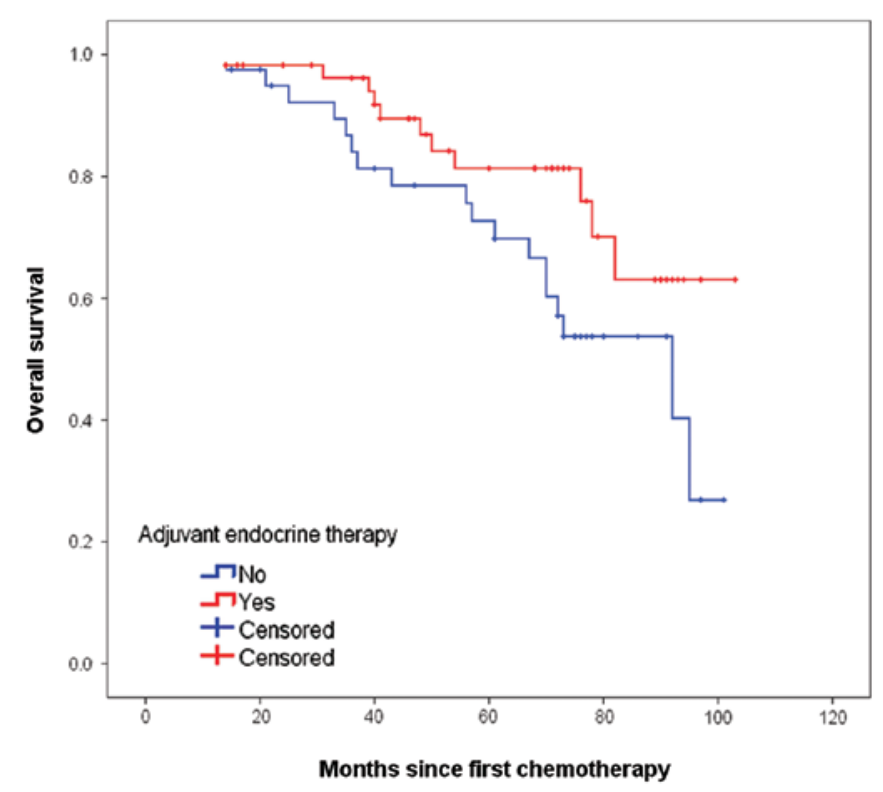

Figure 2. Correlation between the use of ET and OS in all the patients $(\mathrm{P}=0.053)$. ET, endocrine therapy; OS, overall survival.

HER2 status, administration of ET was also associated with an improved DFS, with P-values of 0.013 and 0.122 in the cases of HER2-positive and -negative disease, respectively (Figs. 3 and 4). The other results of univariate analysis are summarized in Table III. The factors associated with an inferior DFS were pre-NAC HER 2 positivity, higher clinical stage, involvement of lymph nodes and omission of ET $(\mathrm{P}<0.05)$. Similarly, higher clinical stage and involvement of lymph node were associated with an inferior OS $(\mathrm{P}<0.05)$.

Multivariate analysis. The results of the multivariate stepwise Cox regression analysis of DFS and OS are shown in Table III. The following variables were included as probable prognostic factors in the Cox proportional hazard model: Age ( $<50$ vs. $>50$ years), menopausal status (premenopausal vs.

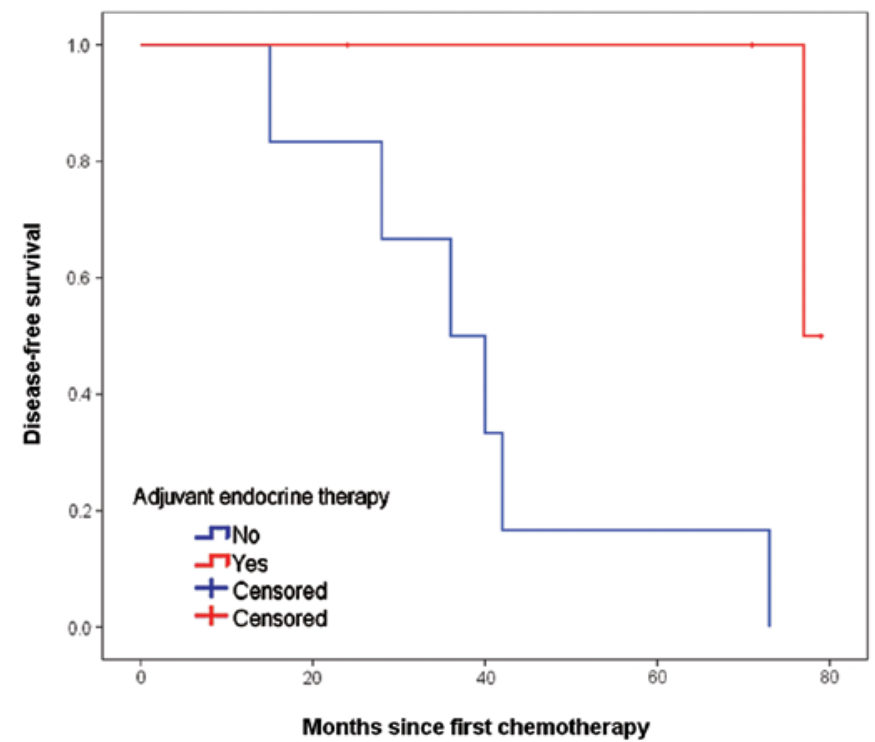

Figure 3. Correlation between the use of ET and DFS in patients with HER2-positive breast cancer $(\mathrm{P}=0.013)$. ET, endocrine therapy; DFS, disease-free survival; HER2, human epidermal growth factor receptor 2.

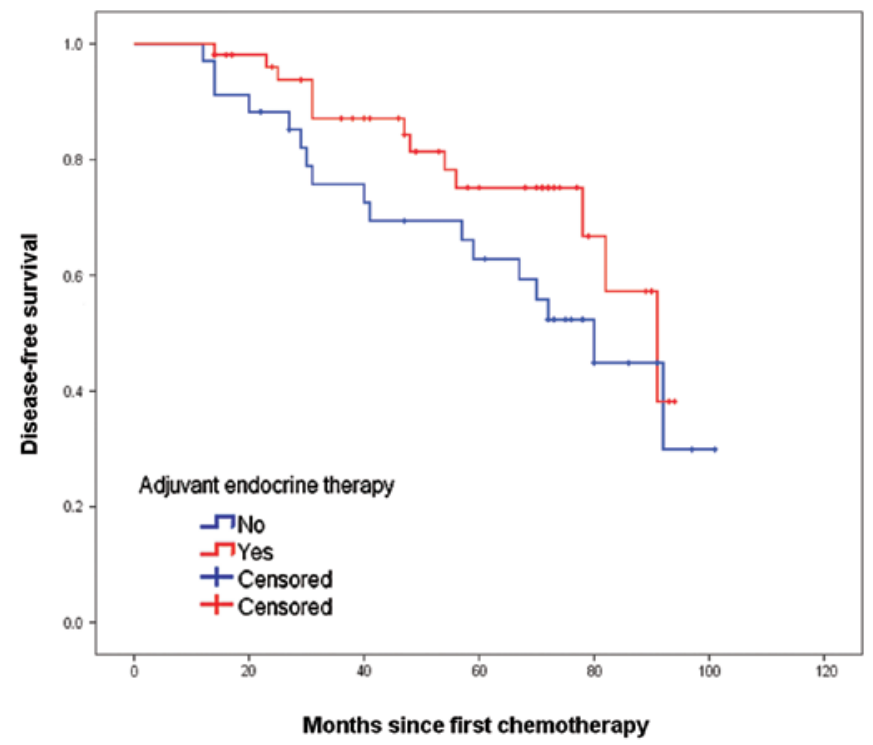

Figure 4. Correlation between the use of ET and DFS in patients with HER2-negative breast cancer $(\mathrm{P}=0.122)$. ET, endocrine therapy; DFS, disease-free survival; HER2, human epidermal growth factor receptor 2 .

postmenopausal), pre-NAC HER2 status (positive vs. negative), NAC regimen (NE vs. CEF vs. ED vs. others), NAC cycle (3-4 vs. 5-6), initial clinical stage (IIb vs. IIIa vs. IIIb-IIIc), clinical tumor response (CR vs. PR vs. SD+PD), pathological axillary lymph node status (positive vs. negative) and adjuvant therapies (ET, chemotherapy and radiation therapy; yes vs. no). Four of these variables (pre-NAC HER2 status, initial clinical stage, pathological axillary lymph node status and the use of adjuvant ET) were identified as the independent predictors for DFS by the stepwise Cox regression model. Similarly, three of these variables (pre-NAC HER2 status, initial clinical stage and pathological axillary lymph node status) were identified as the variables affecting the OS. 
Table I. Patient characteristics, stratified by the use of adjuvant endocrine therapy.

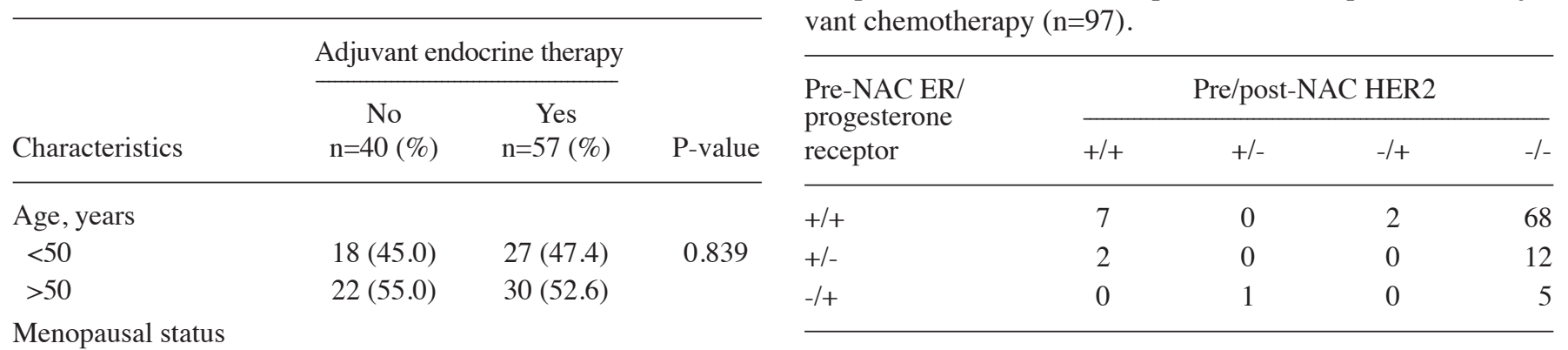

Menopausal status

Premenopausal

Postmenopausal

$23(57.5) \quad 31(54.4)$

0.837

$17(42.5) \quad 26(45.6)$

Pre-NAC ER

\section{Positive}

Negative

$\begin{array}{cc}38(95.0) & 53(93.0) \\ 2(5.0) & 4(7.0)\end{array}$

1.000

Pre-NAC

progesterone receptor

Positive
Negative
Pre-NAC HER
Positive
Negative
NAC
NE
CEF
ED
Others

NAC cycles

$$
\text { 3-4 }
$$$$
5-6
$$$$
35 \text { (87.5) }
$$$$
5(12.5)
$$$$
48(84.2)
$$$$
9 \text { (15.8) }
$$$$
6(15.0)
$$$$
4(7.0)
$$$$
34(85.0)
$$$$
53(93.0)
$$$$
16(40.0)
$$$$
21(36.8)
$$$$
12(30.0)
$$$$
23 \text { (40.4) }
$$$$
6(15.0)
$$$$
8 \text { (14.0) }
$$$$
6 \text { (15.0) }
$$$$
5 \text { (8.8) }
$$

Initial clinical stage

$\begin{array}{llll}\text { IIb } & 10(25.0) & 20(35.1) & 0.575 \\ \text { IIIa } & 16(40.0) & 21(36.8) & \\ \text { IIIb-IIIc } & 14(35.0) & 16(28.1) & \end{array}$

Clinical tumor

$$
\text { response }
$$

\begin{tabular}{lccc} 
CR & $5(12.5)$ & $15(26.3)$ & 0.248 \\
PR & $25(62.5)$ & $28(49.1)$ & \\
SD+PD & $10(25.0)$ & $14(24.6)$ & \\
pALN & & & \\
Positive & $26(65.0)$ & $42(73.7)$ & 0.377 \\
Negative & $14(35.0)$ & $15(26.3)$ & \\
Adjuvant & & & \\
chemotherapy & & & \\
Yes & $37(92.5)$ & $50(87.7)$ & 0.517 \\
No & $3(7.5)$ & $7(12.3)$ & \\
Adjuvant radiation & & & \\
therapy & & & \\
Yes & $32(80.0)$ & $45(78.9)$ & 1.000 \\
No & $8(20.0)$ & $12(21.1)$ & \\
\hline
\end{tabular}

NAC, neoadjuvant chemotherapy; ER, estrogen receptor; HER2, human epidermal growth factor receptor 2; NE, vinorelbine, epirubicin; $\mathrm{CEF}$, cyclophosphamide, epirubicin, fluorouracil; ED, epirubicin, docetaxel; $\mathrm{CR}$, complete response; PR, partial response; SD, stable disease; PD, progressive disease; pALN, pathological axillary lymph node.
Table II. Number of patients classified by ER, progesterone receptor and HER2 statuses prior and subsequent to neoadjuvant chemotherapy $(n=97)$.

ER, estrogen receptor; HER2, human epidermal growth factor receptor 2; NAC, neoadjuvant chemotherapy.

\section{Discussion}

The discordance in hormone receptor and HER2 status between CNB and excision specimens has been reported in numerous retrospective studies with inconsistent results. A meta-analysis by Chen et al (8) confirmed the high diagnostic accuracy of CNB in evaluating hormone receptor and HER2 status compared to open-excision biopsy in breast cancer patients without NAC. The meta-analysis by Zhang et al (9) concluded that in the patients receiving NAC, the hormone receptor status was significantly altered by the chemotherapy and re-evaluation of the hormone receptor status following NAC should be considered. Regarding the high accuracy of CNB in evaluating hormone receptor status, this type of discordance is primarily caused by NAC.

A small number of retrospective studies have reported the discordances in the hormone receptor status to range from $8-33 \%$ (3-6), and the reported changes are equally distributed between a positive and negative switch in the hormone receptor status. Therefore, ET may be significant in patients with a negative-to-positive switch of hormone receptor status and more attention was administered to the effect of ET in patients with hormone receptor status changed from positive to negative in the present study. To the best of our knowledge, the present study is the first to focus on the effect of ET in patients who exhibited a positive-to-negative switch in the hormone receptor status following NAC. In the study, the long-term benefit of ET in this subpopulation of patients was confirmed. The 5-year DFS rates in ET-administered were significantly higher than that of the ET-naïve patients (77.0 vs. 55.5\%), whereas the 5-year OS rates had a non-statistically significant increase subsequent to the use of ET (81.3 vs. $72.7 \%$ ). Similar results have been reported in a previous study of 59 patients who showed hormone receptor status conversion following NAC; the DFS of 47 ET-administered was significantly longer compared to the 12 ET-naïve patients (HR, $0.19,95 \%$ CI: 0.06-0.60, P<0.004) (4). However, the study only included 30 patients with a positive-to-negative change of the hormone receptor status and all those ET-naïve patients exhibited a negative-to-positive change.

The mechanisms for the change in the hormone receptor status in breast cancer caused by chemotherapy are complicated. Chemotherapy has been indicated to be able to target chemosensitive tumor cells (such as hormone receptor-negative cells) and leave the insensitive tumor cells with a different biology 
Table III. Univariate and multivariate prognostic analysis of DFS and OS.

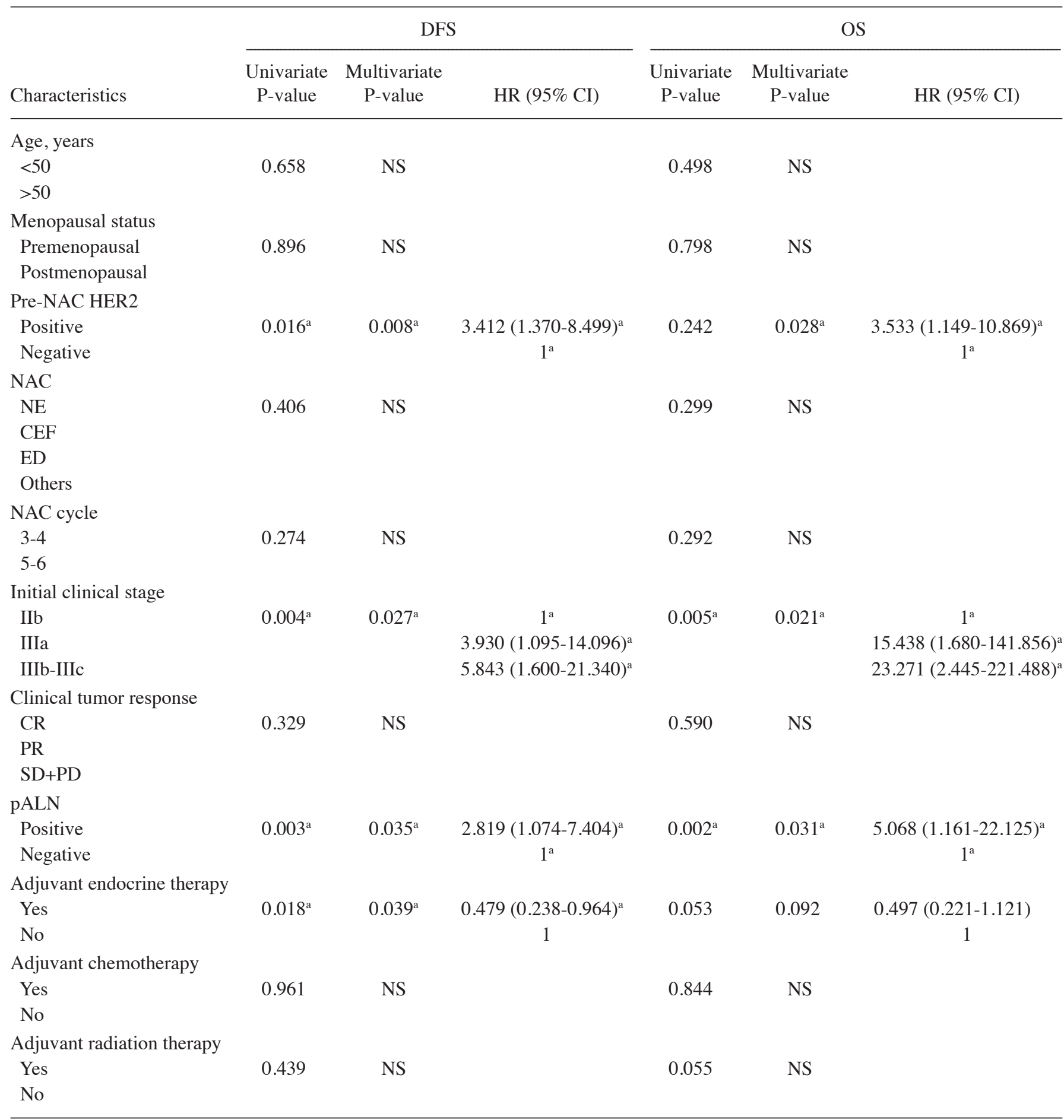

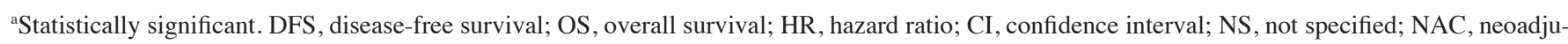
vant chemotherapy; HER2, human epidermal growth factor receptor 2; NE, vinorelbine, epirubicin; CEF, cyclophosphamide, epirubicin, fluorouracil; $\mathrm{ED}$, epirubicin, docetaxel; $\mathrm{CR}$, complete response; PR, partial response; SD, stable disease; PD, progressive disease; pALN, pathological axillary lymph node.

(such as hormone receptor-positive cells) behind in the residual disease, leading to a negative-to-positive change of the hormone receptor. Another possible explanation for a negative-to-positive change of the hormone receptor may be the heterogeneity of the breast carcinoma, as well as the insufficiency of CNB material. The mechanism of the positive-to-negative switch of the hormone receptor status during NAC is more complex. The critical review by van de Ven et al (10) hypothesized that this could be the result of lowered circulating levels of estrogens caused by ovarian insufficiency during chemotherapy, which may cause downregulation of the ER and/or progesterone receptor of the tumor leading to estrogen-independent growth. This is, at least in part, a plausible explanation for the clear downregulation of the hormone receptor following NAC. The 
present study revealed that this type of breast cancer responded to ET, regardless of the underlying mechanism.

With regards to a recent International Expert Consensus, breast cancer can be divided into four intrinsic subtypes on the basis of ER, progesterone receptor, HER2 and Ki67 status (11). Patients with different subtypes of breast cancer exhibit distinct responsiveness to systemic treatment, as well as diverse outcomes (12-14). All the patients in the present study had luminal breast cancer prior to NAC and basal-like or HER2-positive breast cancer following surgery. The results indicated that although the breast cancer was apparently endocrine non-responsive (including basal-like or HER2-positive subtypes) subsequent to surgery, breast cancers with a luminal subtype that was confirmed prior to NAC act like hormonal responsive breast cancer and had a favorable prognosis with the use of ET. Furthermore, luminal breast cancers have been reported to exhibit varied sensitivity to ET, which may be largely, but not exclusively, affected by HER2 status. The multivariate analysis indicated that the benefit of ET was independent of the HER2 status. HER2-positive breast cancers appeared to be more responsive than HER2-negative ones. Due to the limited sample size of the subgroup analysis, this result required further study in a large cohort of patients.

In conclusion, the present study demonstrated that the hormone receptor status should be evaluated not only in the CNB specimens obtained prior to NAC, but also in specimens obtained during post-NAC surgery. The mechanism of the positive-to-negative switch of the hormone receptor status during NAC remains unclear, however, ET is warranted in patients with a pre-NAC-positive hormone receptor status, even though it becomes negative postoperatively.

\section{Acknowledgements}

The authors would like to thank the patients and family members for their willingness to cooperate in the present study. The study was supported in part by grants from the Leading Academic Discipline Project of Shanghai Municipal Education Commission (project no. J50208).

\section{References}

1. Berruti A, Generali D, Kaufmann M, Puztai L, Curigliano G, Aglietta M, Gianni L, Miller WR, Untch M, Sotiriou C, et al: International expert consensus on primary systemic therapy in the management of early breast cancer: highlights of the Fourth Symposium on Primary Systemic Therapy in the Management of Operable Breast Cancer, Cremona, Italy (2010). J Natl Cancer Inst Monogr 2011: 147-151, 2011.
2. Kaufmann M, von Minckwitz G, Mamounas EP, Cameron D, Carey LA, Cristofanilli M, Denkert C, Eiermann W, Gnant M, Harris JR, et al: Recommendations from an international consensus conference on the current status and future of neoadjuvant systemic therapy in primary breast cancer. Ann Surg Oncol 19: 1508-1516, 2012.

3. Burcombe RJ, Makris A, Richman PI, Daley FM, Noble S, Pittam M, Wright D, Allen SA, Dove J and Wilson GD: Evaluation of ER, PgR, HER-2 and Ki-67 as predictors of response to neoadjuvant anthracycline chemotherapy for operable breast cancer. Br J Cancer 92: 147-155, 2005.

4. Hirata T, Shimizu C, Yonemori K, Hirakawa A, Kouno T, Tamura K, Ando M, Katsumata N and Fujiwara Y: Change in the hormone receptor status following administration of neoadjuvant chemotherapy and its impact on the long-term outcome in patients with primary breast cancer. Br J Cancer 101: 1529-1536, 2009.

5. Jain V, Landry $M$ and Levine EA: The stability of estrogen and progesterone receptors in patients receiving preoperative chemotherapy for locally advanced breast carcinoma. Am Surg 62: 162-165, 1996.

6. Tacca O, Penault-Llorca F, Abrial C, Mouret-Reynier MA, Raoelfils I, Durando X, Achard JL, Gimbergues P, Curé H and Chollet P: Changes in and prognostic value of hormone receptor status in a series of operable breast cancer patients treated with neoadjuvant chemotherapy. Oncologist 12: 636-643, 2007.

7. Eisenhauer EA, Therasse P, Bogaerts J, Schwartz LH, Sargent D, Ford R, Dancey J, Arbuck S, Gwyther S, Mooney M, et al: New response evaluation criteria in solid tumours: revised RECIST guideline (version 1.1). Eur J Cancer 45: 228-247, 2009.

8. Chen X, Yuan Y, Gu Z and Shen K: Accuracy of estrogen receptor, progesterone receptor, and HER 2 status between core needle and open excision biopsy in breast cancer: a meta-analysis. Breast Cancer Res Treat 134: 957-967, 2012.

9. Zhang N, Moran MS, Huo Q, Haffty BG and Yang Q: The hormonal receptor status in breast cancer can be altered by neoadjuvant chemotherapy: a meta-analysis. Cancer Invest 29: 594-598, 2011.

10. van de Ven S, Smit VT, Dekker TJ, Nortier JW and Kroep JR: Discordances in ER, PR and HER2 receptors after neoadjuvant chemotherapy in breast cancer. Cancer Treat Rev 37: 422-430, 2011.

11. Goldhirsch A, Wood WC, Coates AS, Gelber RD, Thürlimann B and Senn HJ; Panel members: Strategies for subtypes - dealing with the diversity of breast cancer: highlights of the St. Gallen International Expert Consensus on the Primary Therapy of Early Breast Cancer 2011. Ann Oncol 22: 1736-1747, 2011.

12. Perou CM, Sørlie T, Eisen MB, van de Rijn M, Jeffrey SS, Rees CA, Pollack JR, Ross DT, Johnsen H, Akslen LA, et al: Molecular portraits of human breast tumours. Nature 406: 747-752, 2000.

13. Sørlie T, Perou CM, Tibshirani R, Aas T, Geisler S, Johnsen H, Hastie T, Eisen MB, van de Rijn M, Jeffrey SS, et al: Gene expression patterns of breast carcinomas distinguish tumor subclasses with clinical implications. Proc Natl Acad Sci USA 98: 10869-10874, 2001.

14. Chen XS, Wu JY, Huang O, Chen CM, Wu J, Lu JS, Shao ZM, Shen ZZ and Shen KW: Molecular subtype can predict the response and outcome of Chinese locally advanced breast cancer patients treated with preoperative therapy. Oncol Rep 23: 1213-1220, 2010 